\title{
Response to Lacassie
}

However, the two systems are not identical in purpose or execution. While both systems intend to clear the muddy waters of specifying the diagnoses of patients, the emphasis of our system was to resolve the dichotomy facing the field of genetics today. Do we define a condition by its molecular genetic pathogenesis, or by the observation of specific clinical findings? As was discussed in a recent commentary in Science, ${ }^{3}$ this debate will intensify as more disease-related gene alterations are determined. As we reviewed in our paper, ${ }^{1}$ there are numerous examples that clearly show the "either-or" approach of molecular versus clinical descriptors does not work. Our nomenclature system was an attempt to integrate clinical and molecular descriptors. As such, the impetus behind our proposal was to assist researchers and clinicians to speak the same language. The benefits to clinical diagnosis for a specific patient derive from that primary focus.

Although our primary goal was to unify the nomenclature system of clinicians and molecular biologists to facilitate research and patient care, the system has already demonstrated the potential to assist practicing clinical geneticists in another important arena, namely, reimbursement. To that end, we have begun working with the American College of Medical Genetics to integrate some of the features of the multiaxis system into a comprehensive nomenclature system useful for basic and clinical research, clinical care, and reimbursement.

We stake no claim of originality for the system, feeling that is due to the designers of the DSM. By publishing our proposal in a widely read and indexed journal and following that with discussions with the College, we hope to be facilitate the development of a comprehensive nomenclature system for medical genetics research and clinical practice that will benefit our colleagues and our patients.

\section{Nathaniel H. Robin, MD \\ Center for Human Genetics University Hospitals of Cleveland Case Western Reserve University School of Medicine Cleveland, Ohio}

To the Editor:

We appreciate Dr. Lacassie's comments and his support for a nomenclature system for medical genetics. ${ }^{1}$ Like us, Dr. Lacassie's devised a genetic diagnostic system ${ }^{2}$ based on a simple modification of the Diagnostic and Statistical Manual of Mental Disorders (DSM). We missed his citations in our original literature searches as none of them are included in Medline. Our error arose when a reviewer from a prior submission of this manuscript to another journal pointed out one of these citations. We forgot to include that citation when the paper was reformatted for submission to this journal and we regret that omission.

Leslie G. Biesecker, MD

Genetic Diseases Research Branch National Human Genome Research Institute Bethesda, Maryland

\section{References}

1. Robin NH, Biesecker LG. Considerations for a multiaxis nomenclature system for medical genetics. Genet Med 2001;3:290-293.

2. Lacassie Y. An international multiaxial diagnostic system in clinical genetics. In: Bartsocas CS, Beighton P, editors. Dysmorphology and genetics of cardiovascular disorders. Athens: Zerbinis, 1994:28-31.

3. Temple LKF, McLeod RS, Gallinger S, Wright JG. Defining disease in the genomics era. Science 2001;293:807-808. 